\title{
Active Probe Emitting HF Electromagnetic Fields and Piezoelectric Emissions in Association with Local Carbon Nanotubes : Preliminary Tests in C6 Glioma Cell Death Induction: A Possible Application in Glioblastoma Therapeutics?
}

\author{
Debouzy JC ${ }^{1-3 *}$, Crouzier $\mathbf{D}^{1-3}$, Ballester $\mathbf{B}^{1,2}$ and Foerster $\mathbf{M}^{2,3}$ \\ ${ }^{1}$ IRBA, Biomedical Research Institute, France \\ ${ }^{2}$ CEA/CLINATEC, Rue M Esclangon, France
}

${ }^{3}$ REMEDEE LABS, chemin du vieux chêne, France

*Corresponding author: Debouzy JC, IRBA, Biomedical Research Institute, France

\section{ARTICLE INFO}

Received: 慧 February 04, 2019

Published: 幽 February 13, 2019

Citation: Debouzy JC, Crouzier D, Ballester B, Foerster M. Active Probe Emitting HF Electromagnetic Fields and Piezoelectric Emissions in Association with Local Carbon Nanotubes : Preliminary Tests in C6 Glioma Cell Death Induction: A Possible Application in Glioblastoma Therapeutics?. Biomed J Sci \& Tech Res 14(3)-2019. BJSTR. MS.ID.002563.

\begin{abstract}
Glioblastoma are malignant tumors of the central nervous system with generally a lethal issue within 15 months whatever the classical therapeutics. Several studies showed promising results using alternative electric or magnetic fields, or nanoparticles (mainly iron) combined with an alternative magnetic field. Besides, Carbon Nanotubes (CNTs) have very special physicochemical properties potentially synergistic with non-ionizing radiation (EMF, including ultrasound from a piezo component) exposure. The aim of this first note was to the effects of a co-exposition from carbon nanotubes and electromagnetic field on an in vitro $\mathrm{C} 6$ glioma model growth and viability, using model probe associating EMF (100 KHz, square signal, 10Vpp) and piezo emission with a local CNTs deposit. A strong synergic effect between EMF and CNTs as the C6 cell population decrease with these two factors. Respectively, $10 \%, 25 \%$ and $51 \%$ population decrease for the Sham / Field, Sham / Nano and double exposed group compared to the control group. However, the survival rate of the cells is poorly affected after $72 \mathrm{~h}$ exposure time (more than $90 \%$ survival rate). These promising results should be used to a miniaturized implantable probe center in order to propose a new therapeutic approach using an intra tumoral catheter (including an EMF emitter), allowing the distribution of CNTs (or chemotherapy) using the convective infusion technique.
\end{abstract}

Keywords: HF; RF; Piezo; Carbon Nanotubes; Glioma Therapeutics

\section{Introduction}

Malignant Glioma (MGs) variants, Glioblastoma (GBM) is the most common malignant tumor in the central nervous system. His prevalence is about $1 / 100000$, with a survival time post-diagnosis of 15 months and a mortality rate exceeding 95\% [1]. Common treatments for GBM include surgical resection, radiotherapy, chemotherapy, or combinations of these three modalities [2,3]. Nevertheless, there is no general agreement on the radiation pro tocol to use. If fractionation seems to be the most appropriate, the choice mainly depends on the radiotherapy options available to the treating physician. In case of recurrent glioblastoma, the treatment is then focused on the use of chemotherapy. Numerous studies have demonstrated the safety and efficiency of various agents, both alone and in combination [4-8]. GBM have an extremely low survival rate due to the high infiltrating properties of the glioma cells. Typically, 
these cells could infiltrate up to $2 \mathrm{~cm}$ exceeding the volume of visible tumor, making them difficult to detect and treat. Treatment of GBM is also limited by several other factors such as, the insufficient delivery of chemotherapy drugs caused by the blood-brain-barrier, the radio resistance of the cells and the need to preserve functional parenchyma.

Despite the existence of different therapeutic strategy treatments, the evolution of the post-diagnosis median survival only increased from 13 to 15 month, highlighting the need of curative approaches. In the recent past, some new innovative researches have produced promising results or are the subject of ongoing investigations [9-16]. Among them, we can find biophysics approaches using electromagnetic fields or nanoparticles. The results of Kirson's group highlighted the first point. This team have shown a decrease of the malignant cell proliferation with no or minimal side effects in animal tumor models and human brain tumors [11.12] by using an alternative electric field (also called TT Fields for Tumor Treating Field). According to the authors, the mechanism involved is related to the disintegration of the mitotic spindle [11]. NovoTTF therapy is a new therapy using this observation and has undertaken the phase III clinical trial [13].

Another Group (Magforce AG) is using iron nanoparticles combined with an alternative magnetic field (100 kHz). This field induces nanoparticle's vibrations and then, locally increases the temperature leading to the tumor cells death. The main drawback of such approach is the lack of specificity. Even if nanoparticles are addressed to malignant cells, the increase in temperature destroys all the cells located in the vicinity of the particle. However, this therapy seems efficient and safe with no or moderate side effects [14-16]. We propose an alternative project using these two different aspects by using both nanoparticles and electromagnetic field without inducing any thermal effect. This new approach is inspired by recent works [17] showing a strong interaction between Carbon Nanotubes (CNTs) and electromagnetic field.

According to this observation, a synergic toxic effect between electromagnetic fields (RF) and CNTs could be expected. CNTs could increase the toxicity of the RF by focusing them and inducing an electromagnetic field increase. The RF could also increase the toxicity of the CNTs by a direct helping of their dispersion and penetration into the cells. The intrinsic characteristics of CNTs make them specifically sensitive to electromagnetic field. Due to theirs high length compared to their diameter, they could be at the origin of a strong point effect (like a lightning rod) inducing a local increase of the fields' strength. Both CNTs and electromagnetic field are major public health issues and many studies tried to determinate their toxicity [18-21].

The drawbacks of both Kirson and Magforce AG studies was that the field emitter (electrodes on the skin / external magnetic emitter) were located outside the body, i.e. far away from the target. As field power density generally decreases with the square of the distance, using an intra cerebral emitter, combined with CNTs induced local magnification of the field would be promising. Such mechanisms would clearly differ from those of Magforce AG team, since neither tissue heating nor thermal reaction occur in the former case. Final therapeutic hope would use a combination of the convective infusion technique $[22,23]$ together with the inclusion of the RF emitter in the catheter used for intra-tumoral injection. The aim of this study was to evaluate the effects of a co-exposure to carbon nanotubes and combined electromagnetic field/ultrasounds on an in vitro glioma model.

\section{Materials and Methods}

\section{Cell Culture}

The C6 cells (Rat Glioma) were obtained from the ATCC cell base (ATCC $®$ CCL-107 $7^{\mathrm{TM}}$ ). The cells were maintained in exponential growth in a $5 \% \mathrm{CO} 2$ humidified incubator held at $37^{\circ} \mathrm{C}$. The $\mathrm{C} 6$ cells were placed in cell culture flasks $\left(75 \mathrm{~cm}^{2}\right)$ and were grown with 12 $\mathrm{ml}$ of DMEM, Glutamax区 supplemented with $10 \%$ heat-inactivated fotal bovine serum and antibiotics $(100 \mathrm{U} / \mathrm{ml}$ penicillin and $100 \mu \mathrm{g} / \mathrm{ml}$ streptomycin).

\section{Synthesis and Particles Characterisation}

Single wall carbon nanotubes (CNTs, quoted here CNTs, Figure 1) produced by Catalytic Chemical Vapor Deposition were purchased by SIGMA-Aldrich, St Quentin Fallavier, France. The CNTs diameter distribution was ranged from 0.7 to $1.3 \mathrm{~nm}$, and length between 450 and $2300 \mathrm{~nm}$, although bundles may be much longer (up to $100 \mu \mathrm{m}$ at least) with a density of $1.7-1.9 \mathrm{~g} / \mathrm{cm} 3$ at $25^{\circ} \mathrm{C}$.

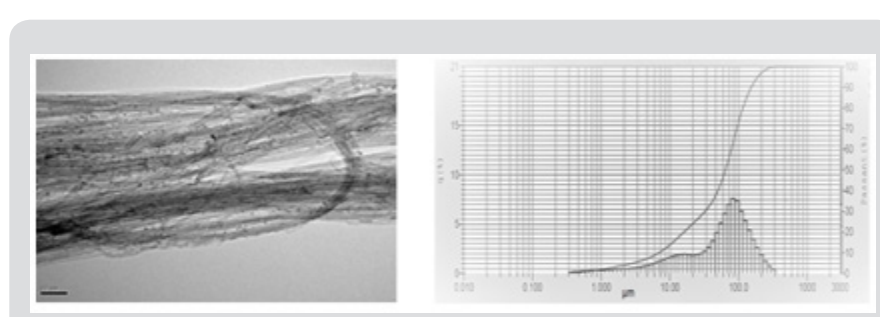

Figure 1: Left : Electron microscope of CNTs in suspension in aqueous medium after dispersion Left : relative distribution of CNTs aggregates in suspension.

Particle size distribution of the stock solution used for cells exposure was analysed with a Laser Scattering Particle Size Distribution Analyser, LA-950V2 (HORIBA) with DMEM as solvent. Particles dispersion in cell culture medium and cells exposure : Stock solution of $5 \mathrm{mg} / \mathrm{ml}$ CNTs was prepared by dispersion of sterilised particles in DMEM containing 25 \% FBS. Previous study has reported that serum produced particle suspensions were observed with the fewest large agglomerates [24]. The nanoparticles were homogeneously dispersed by vortexing for 30 seconds followed by sonication in a water bath (Al 04-02, Advantage Lab) for $5 \mathrm{~min}$. This procedure was repeated five times. A "Sham Stock Solution" of sterile DMEM containing $25 \%$ FBS was prepared and sonicated at the same time for use as control. 
The stock solutions were kept at $4{ }^{\circ} \mathrm{C}$. The stock solution of CNTs was resuspended by vortexing for 30 s followed by sonication for 5 min and diluted in cell culture medium to the required concentration $(50 \mu \mathrm{g} / \mathrm{ml}, 120 \mu \mathrm{l}$ per flask). An equal volume of "Sham Stock Solution" was added to cell culture medium for control cells. Before any experiment, CNTs size were analyzed using a Laser Scattering Particle Size Distribution Analyser, LA-950V2 (HORIBA) with DMEM as solvent $(10 \%)$ and a dilution at one centesimal $(150 \mu \mathrm{l}$ in $15 \mathrm{ml}$ ). The size of the CNTs measured by light scattering was defined as the equivalent spherical diameter and could not be related to the exact particle size but represent a relative size of nanotubes which are long cylinders. This led to identify different particle sizes in solution : $100 \mu \mathrm{m}$ diameter aggregates (35\%), a minor component of intermediate aggregates $(1 \mu \mathrm{m}-30 \mu)$. Sizes below $1 \mu$ were considered as unbounded carbon nanotubes.

\section{Temperature Control}

The absence of temperature increase was controlled over $16 \mathrm{Hrs}$ exposure under the RF/CNTs conditions with probe immersed in the culture flask, using a FLIR A320 Temp screen camera. No significant variation was detected within the observation period.

\section{Electromagnetic Probe}

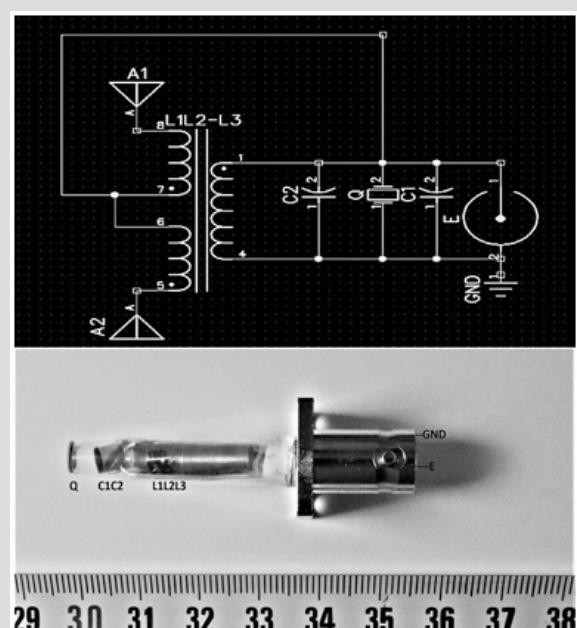

Figure 2: Top: Electronic wiring of the probe; Bottom: The probe, as connected with BNC connectors. GND: Ground E: RF input via BNC connector; Q: $100 \mathrm{KHz}$ resonating piezo; $\mathrm{C} 1, \mathrm{C} 2$ tune capacitors; $\mathrm{L} 1=1 \mathrm{mH}, \mathrm{L} 2=\mathrm{L} 3=16 \mathrm{mH}$; L2L3-L1transformator; A1, A2: antennas.

Measurement of the electromagnetic field : The probe was composed of three parts (electric, magnetic and ultrasonic with a piezoelectric component, CNTs) realizing the emission of an electromagnetic signal and a piezoelectric operating phase. Electromagnetic fields were then applied by connecting the wires to an amplifier operated by a signal generator with frequency and amplitude controls. The probe was powered by a square wave modulated signal working at $100 \mathrm{kHz}$ (low-intermediate frequency emission) from an Agilent 3312DA generator, peak to peak tension of $10 \mathrm{Vpp}$, and duty cycle $\mathrm{DC}=50 \%$. This first prototype version is presented Figure 2.

Field estimations were attempted with a PM03 field meter and a H/E fieldmeter ESM-100, Meshket. As the exposure conditions (inside the flask) were obviously in reactive nearfield conditions, E values were extremely heterogenous $(5 \mathrm{~dB}$, with a maximum of $80 \mathrm{~V} / \mathrm{m}$ in close vicinity of the probe extremity ) while $\mathrm{H}$ variations were found less important ( $3 \mathrm{~dB}$, maximum $90 \mathrm{nT}$ in close vicinity of the probe extremity). The $100 \mathrm{kHz}$ resonating piezo was a PRYY0073 from PICERAMIC, Germany. The electric field was evaluated with the help of a PMOR 03 field meter. In order to isolate the exposed flask, the electromagnetic exposed flask was placed into an absorbing chamber allowing an attenuation of the signal by $12 \mathrm{~dB}$.

\section{In vitro Experimental Setup}

Each flask was randomly assigned to one of the four different groups: Sham / Sham, Sham / Nano exposed, Sham / Field exposed, and Nano / Field exposed (CNTs/RF+piezo, quoted CNTs/RF in the followings). All the cultures were realized in triplicate under the same conditions as the CNTs/RF group except that the generator was turned off, with the probe into the flask. The initial C6 cell population was $0.2 * 106$ cells per flask. $72 \mathrm{~h}$ exposure starts immediately after the cells have been passaged. Sham / Sham and Sham / Field groups received $120 \mu \mathrm{l}$ of the Stock Sham solution (without CNTs), the double exposed and the Sham / Nano groups received $120 \mu \mathrm{l}$ of the Stock solution containing the CNTs. After exposure, in order to eliminate the SWCNTs in solution, each flask was rinsed 3 times with Phosphate Buffered Saline (PBS). The cells were chemically detached from the flask using trypsin EDTA. C6 cells were centrifuged $5 \mathrm{~min}$ at 300G. Cell pellets were resuspended in $1 \mathrm{ml}$ cell culture medium. $50 \mu \mathrm{l}$ of C 6 cells solution were added to $50 \mu \mathrm{l}$ of Trypan Blue. C6 cells were counted by a TC20TM automatic cell counter. Results were expressed as means \pm Standard Deviation (S.D.)

\section{Results}

\section{Cells Count and Viability}

In these experiments, cell counts were performed after $72 \mathrm{~h}$ culture with/without RF exposure and/or in the presence or not of CNTs (see Figure 3). By comparison with reference sham values measured at $72 \mathrm{~h}(2,84106 / \mathrm{mL})$ and also after $72 \mathrm{Hr}$ RF exposure $(2,8106 / \mathrm{mL})$ an important reduction in absolute values of cells counts was found when CNTs were present alone $\left(2.10^{6} / \mathrm{mL}\right)$ and especially when CNTs were associated with RF exposure $(1,46$ $106 / \mathrm{mL}$ ) . These results are all the more evident when expressed in percentage to reference sham values and showed an important decrease in the number of the cells compared to the control group (51\% for the Nano / Field group; 75\% for the Sham / Nano group; 98\% for the Sham / Field group). Conversely, the survival rate of the cells within any group was only slightly decreased whatever the exposition factors considered : hence, the survival rate is maxima (100\%) for the Sham / Sham control group and slowly decreases 
with the exposition of CNTs and electromagnetic field (97\% survival rate for the Sham / Field group, 95\% survival rate for the Sham / Nano group and 94\% survival rate for the Nano / Field group. As this step it is worth to note than rinsing process (see methods) induced an averaging over the whole flask, this resulting in a smoothing of any possible local drastic effect. Morphological control had thus to be performed.

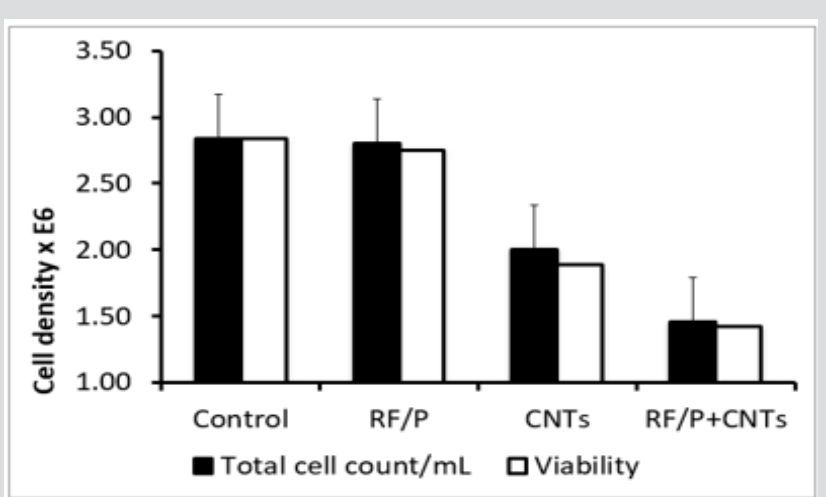

Figure 3: Cell count (cells/ml $10^{6}$ related to sham / sham group (percentage) and survival rate distribution in C6 cell population with exposition of SWCNTs or electromagnetic field according to their respective group. The cells were exposed for $72 \mathrm{~h}$ right after injection of the cells into the flask.

\section{Cell Morphologic Observation}
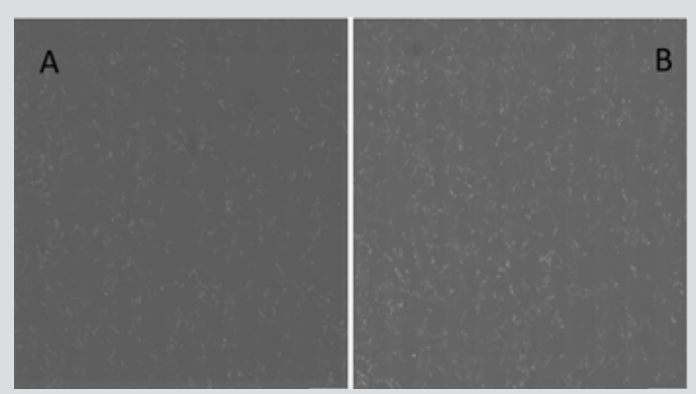

Figure 4: A: Sham / Sham group cells after $72 \mathrm{~h}$ exposure time (X4 magnification); B: CNTs + RF group cells after $72 \mathrm{~h}$ exposure time and rising of the flask ( $\mathrm{X} 4$ magnification).

As mentioned above, microscopic observation (Figure 4) clearly shows the reduction of cell density in the flask, even like here after the rinsing process between CNTs/RF and sham groups. Moreover, the absence of morphological change was also in full agreement with the minor differences in cell viability between groups. Here differences appear between CNTs and CNTs/+RF groups. As noted in the methods section, CNTs spontaneously selforganized in $\mu$-sized aggregates, with only a partial contribution of dispersed nanoparticles. This was observed on the Figure 5A, especially before rinsing of the flasks, where these aggregates are visible even at low magnification ( $\mathrm{x} 4$ ). Such a distribution appeared partially overcome (or really dispersed) due to rinsing, as shown on Figure 5B, where higher magnification allows to detect smaller particles among the $\mathrm{C} 6$ cells, without specific contact or geometric distribution.
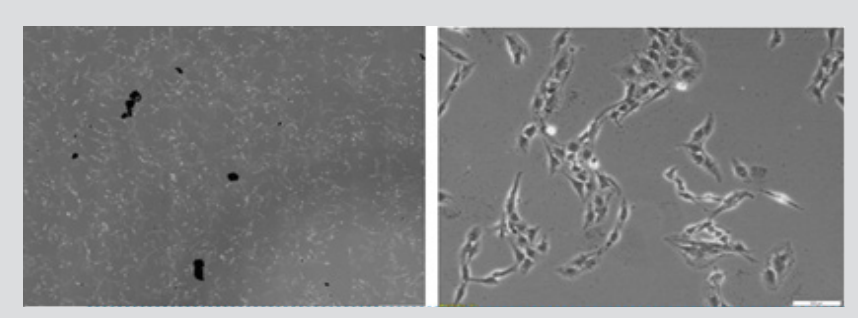

Figure 5: Sham / Nano group cells after 72h exposure time with nanotubes aggregates, before the rinsing process, with X4 magnification (left) and X10 magnification (right).

Besides, no evidence for cell distribution, i.e. heterogeneity around the probe (not emitting, sham) was observed. Such was not the case in the combined CNTs/RF samples (Figure 6). Several CNTs aggregates were still present whereas more numerous and of smaller size, in an area close to the extremity of the probe. This feature was not reasonable to quantify - a factor of 2 to 5 ? - due to the important heterogeneity in the half centimeter around the probe wall. However, a true "no cell area" was truly present in the several millimeters around the probe. Furthermore, linear arrangements of CNTs were observed (Figure 6) in the closest vicinity of the antennas (wires inside the probe while aligned along its main axis) , this revealing true interactions between CNTs and RF and/or ultrasound coming from the piezo.

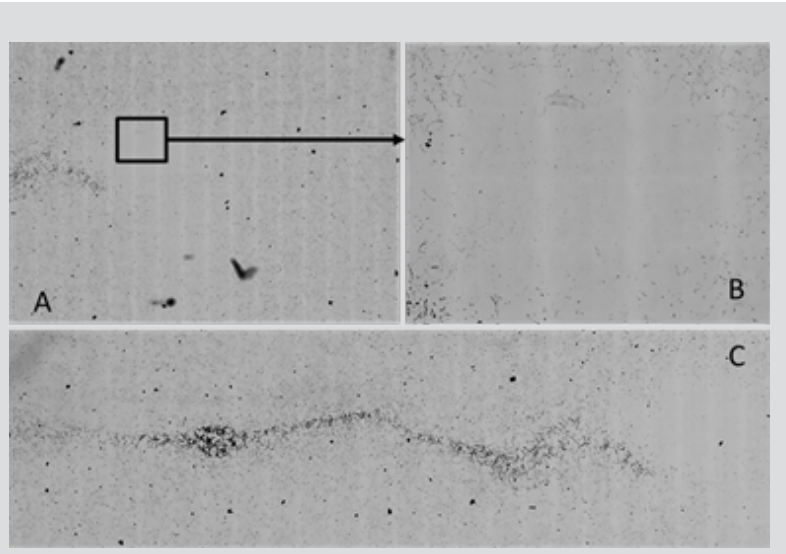

Figure 6: CNTs + RF group after $72 \mathrm{~h}$ exposure time showing a cell depleted area close to the probe extremity; longitudinal CNTs orientation in the CNTS+RF group after $72 \mathrm{~h}$ exposure time before rinsing resulting homogenization.

\section{Discussion}

The aim of the preliminary work was to evaluate the effects of a co-exposition from carbon nanotubes and electromagnetic field on an in vitro glioma model. The Sham / Field group cell count showed a limited decrease of the C6 cells population compared to the Sham / Sham group and a similar survival rate between the two groups, $99 \%$ and $100 \%$ respectively. Compared to Kirson's results several differences arise $(9,11)$ :in the present work, the frequency was fixed at $100 \mathrm{kHz}$ while Kirson's group used a 100-300 kHz frequency system, with an emitting power twice as ours. The Sham / CNTs 
group cell count showed a $25 \%$ decrease of the C6 cells population compared to the Sham / Sham group with a $97 \%$ survival rate. CNTs alone have a strong effect on C6 cells. This could be related to their own direct or indirect toxicity related with the ability of CNTs to trap growing factors in a culture medium [18-21]. The most interesting results were to find a synergic effect of the CNTs with the electromagnetic field. The Nano $+\mathrm{RF}$ cell count showed decrease to $51 \%$ of the C6 cells population compared to the Sham / Sham group with a 95\% survival rate. As an hypothesis, the SWCNTs could induce an increase of the local field's strength and a disruption of the field direction. According to the mechanism of action of TTFields described by Kirson, TTField efficacy must be a function of the angle between the field and axis of division of the cells; when the two are parallel it has a maximal effect and when one is perpendicular to the other, it has a minimal effect [11].

In our case, the carbon nanotubes could solve this problem as they might cause a local reorientation of the field, overcoming the previous problem. In addition, the effect of the piezoelectric component might be useful to open the blood-brain-barrier and increase the dispersion of the CNTs into the tumor after a local injection using the convective infusion technique [23]. The results showed a strong effect associated with an important decrease of the C6 population subjected to the double exposure. However, the survival rate of the cells is still high and this combination tends to prove that the effect of the co-exposition is not on the destruction of the cells herself but by an inhibition of the cell proliferation [11].

At this step it is not possible to ensure any specific effect due to the several bias of the study. First of all, the studied population of cell wasn't enough to draw conclusion. More experimentation should be performed in order to obtain a strong statistical analysis. In addition, most of the dead cells are eliminated during the rising process because they can't bind the flask anymore once they lost their structure. In order to include these cells into the final calculation, centrifugation of the rising solvent might be a good idea to retrieve dead cells. Another possibility would be gel phase culture and direct final count by optical density lecture, for instance. Besides, the specific effect of each component separately or combined with one or all the others should be addressed, for instance separate piezo and RF contribution and association with CNTs. Finally, the particle exposure could also be improved.

The particle size distribution showed that the dispersion of the carbon nanotubes was neither homogenous not ideally dispersed. Due to their strong hydrophobic properties, CNTs bind each other and form large aggregates. This could diminish the efficacy of the CNTs on the cells and hide a potential effect. The CNTs concentration used here $(50 \mu \mathrm{g} / \mathrm{ml})$ may be at the origin of the problem and using a lower concentration could increase the dispersion of the carbon nanotubes. This concentration was chosen based on the previous studies on the CNTs toxicity $[18,21,27]$. The CNTs concentration isn't the only parameter that could affect the results. The previous pictures were obtained using a phase contrast technique that could not distinguish apoptotic cells and necrotic cells (especially at the beginning of an apoptotic process).

Flow cytometry imaging could solve this problem, with a Hoechst stain to visualize the cell nucleus or even a fluorescent stain using PKH $[28,29]$. From a mechanistic point of view, different cell types should be tested to ensure (or not) the existence of aspecific mechanisms at mitotic bundle destructuration, as mentioned by Kirson. From this, and by designing the probe small enough to be implanted in living systems, in vivo experimentation procedures would be envisaged. These different points are presently addressed, and we plan to set up a "matches-sized" probe, including a coaxial catheter for direct perfusion of CNTs or charged drugs. Hence other drugs could be carried by the convective infusion system and the combination with a special drug carried by CNTs [30]. The idea of an intra tumoral catheter including an RF emitter allowing the distribution of chemotherapy or carbon nanotubes using the convective infusion technique seems promising. This thematic actually plans to in vivo animal experimentations procedure where other questions such as possible blood-brain-barrier opening and safety evaluation of such therapy.

\section{Aknowledgment}

Many thanks to Dash and Laure Minier for English correction, and to Drs S Dekali, F Del Vecchio A. Bourgois and A Boyard for particles characterization.

\section{References}

1. Nishikawa R (2010) Standard therapy for glioblastoma - A review of where we are. Neurol Med Chir (Tokyo)50(9): 713-719.

2. Walker MD, Green SB, Byar DP, Alexander E, Batzdorf U, et al. (1980) Randomized comparisons of radiotherapy and nitrosoureas for the treatment of malignant glioma after surgery. N Engl J Med 303(23): 1323-1329.

3. Stupp R, Hegi ME, Mason WP, van den Bent MJ, Taphoorn MJB, et al. (2009) Effects of radiotherapy with concomitant and adjuvant temozolomide versus radiotherapy alone on survival in glioblastoma in a randomised phase III study: 5-year analysis of the EORTC-NCIC trial. Lancet Oncol 10(5): 459-466.

4. Keles GE, Lamborn KR, Chang SM, Prados MD, Berger MS (2004) Volume of residual disease as a predictor of outcome in adult patients with recurrent supratentorial glioblastomas multiforme who are undergoing chemotherapy. J Neurosurg 100(1): 41-46.

5. Brandes AA, Tosoni A, Cavallo G, Bertorelle R, Gioia V, et al. (2006) Temozolomide 3 weeks on and 1 week off as first-line therapy for recurrent glioblastoma: Phase II study from gruppo italiano cooperativo di neuro-oncologia (GICNO). Br J Cancer 95(9): 1155-1160.

6. Brandes AA, Tosoni A, Franceschi E, Blatt V, Santoro A, et al. (2009) Fotemustine as second-line treatment for recurrent or progressive glioblastoma after concomitant and/or adjuvant temozolomide: A phase II trial of Gruppo Italiano Cooperativo di Neuro-Oncologia (GICNO). Cancer Chemother Pharmacol 64(4): 769-775.

7. Wick A, Felsberg J, Steinbach JP, Herrlinger U, Platten M, et al. (2007) Efficacy and tolerability of temozolomide in an alternating weekly regimen in patients with recurrent glioma. J Clin Oncol 25(22): 33573361. 
8. Gwak HS, Youn SM, Kwon AH, Lee SH, Kim JH, et al. (2005) ACNUCisplatin continuous infusion chemotherapy as salvage therapy for recurrent glioblastomas: Phase II study. J Neurooncol 75(2): 173-180.

9. Kirson ED, Gurvich Z, Schneiderman R, Dekel E, Itzhaki A, et al. (2004) Disruption of cancer cell replication by alternating electric fields. Cancer Res 64(9): 3288-3295.

10. Kirson ED, Schneiderman RS, Dbalý V, Tovaryš F, Vymazal J, et al. (2009) Chemotherapeutic treatment efficacy and sensitivity are increased by adjuvant alternating electric fields (TTFields). BMC Med Phys 9(1): 1.

11. Kirson ED, Dbalý V, Tovaryš F, Vymazal J, Soustiel JF, et al. (2007) Alternating electric fields arrest cell proliferation in animal tumor models and human brain tumors. Proc Natl Acad Sci 104(24): 1015210157.

12. Kirson ED, Giladi M, Gurvich Z, Itzhaki A, Mordechovich D, et al. (2009) Alternating electric fields (TTFields) inhibit metastatic spread of solid tumors to the lungs. Clin Exp Metastasis 26(7): 633-640.

13. Stupp R, Kanner A, Engelhard H, Heidecke V, Taillibert S, et al. (2010) A prospective, randomized, open-label, phase III clinical trial of NovoTTF$100 \mathrm{~A}$ versus best standard of care chemotherapy in patients with recurrent glioblastoma. ASCO Meet Abstr 28(18_suppl): LBA2007.

14. Jordan A, Maier-Hauff K (2007) Magnetic nanoparticles for intracranial thermotherapy. J Nanosci Nanotechnol 7(12): 4604-4606.

15. Maier-Hauff K, Rothe R, Scholz R, Gneveckow U, Wust P, et al. (2007) Intracranial thermotherapy using magnetic nanoparticles combined with external beam radiotherapy: Results of a feasibility study on patients with glioblastoma multiforme. J Neurooncol 81(1): 53-60.

16. Maier-Hauff K, Ulrich F, Nestler D, Niehoff H, Wust P, et al. (2011) Efficacy and safety of intratumoral thermotherapy using magnetic iron-oxide nanoparticles combined with external beam radiotherapy on patients with recurrent glioblastoma multiforme. J Neurooncol 103(2): 317-324.

17. Jensen K, Weldon J, Garcia H, Zettl A (2007) Nanotube Radio. Nano Lett 7(11): 3508-3511.

18. Crouzier D, Follot S, Gentilhomme E, Flahaut E, Arnaud R, et al. (2010) Carbon nanotubes induce inflammation but decrease the production of reactive oxygen species in lung. Toxicology 272(1-3): 39-45.

19. Song Y, Li X, Du X (2009) Exposure to nanoparticles is related to pleural effusion, pulmonary fibrosis and granuloma. Eur Respir J 34(3): 559567.

ISSN: 2574-1241

DOI: 10.26717.BJSTR.2019.14.002563

Debouzy JC. Biomed J Sci \& Tech Res

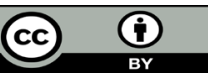

This work is licensed under Creative Commons Attribution 4.0 License

Submission Link: https://biomedres.us/submit-manuscript.php
20. Lindberg HK, Falck GC M, Suhonen S, Vippola M, Vanhala E, et al. (2009) Genotoxicity of nanomaterials: DNA damage and micronuclei induced by carbon nanotubes and graphite nanofibres in human bronchial epithelial cells in vitro. Toxicol Lett 186(3): 166-173.

21. Porter DW, Hubbs AF, Mercer RR, Wu N, Wolfarth MG, et al. (2010) Mouse pulmonary dose- and time course-responses induced by exposure to multi-walled carbon nanotubes. Toxicology 269(2-3): 136-147.

22. Asthagiri AR, Walbridge S, Heiss JD, Lonser RR (2011) Effect of concentration on the accuracy of convective imaging distribution of a gadolinium-based surrogate tracer: Laboratory investigation. J Neurosurg 115(3): 467-473.

23. Lewis GK, Schulz ZR, Pannullo SC, Southard TL, Olbricht WL (2012) Ultrasound-assisted convection-enhanced delivery to the brain in vivo with a novel transducer cannula assembly: Laboratory investigation. J Neurosurg 117(6): 1128-1140.

24. Buford MC, Hamilton RF, Holian A (2007) A comparison of dispersing media for various engineered carbon nanoparticles. Part Fibre Toxicol 4(1): 6 .

25. Ken Donaldson CA P (2013) Pulmonary toxicity of carbon nanotubes and asbestos - similarities and differences. Adv Drug Deliv Rev 65(15): 2078-2086.

26. Oberdorster G, Elder A, Rinderknecht A (2009) Nanoparticles and the Brain: Cause for concern? J Nanosci Nanotechnol 9(8): 4996-5007.

27. Lam CW, James JT, McCluskey R, Hunter RL (2004) Pulmonary toxicity of single-wall carbon nanotubes in mice 7 and 90 days after intratracheal instillation. Toxicol Sci 77(1): 126-134.

28. Vermes I, Haanen C, Reutelingsperger C (2000) Flow cytometry of apoptotic cell death. Flow Cytom 2000 243(1-2): 167-190.

29. Vermes I, Haanen C, Steffens-Nakken H, Reutellingsperger C (1995) A novel assay for apoptosis Flow cytometric detection of phosphatidylserine expression on early apoptotic cells using fluorescein labelled Annexin V. J Immunol Methods. 184(1): 39-51.

30. Wong BS, Yoong SL, Jagusiak A, Panczyk T, Ho HK, et al. (2013) Carbon nanotubes for delivery of small molecule drugs. Adv Drug Deliv Rev 65(15): 1964-2015.

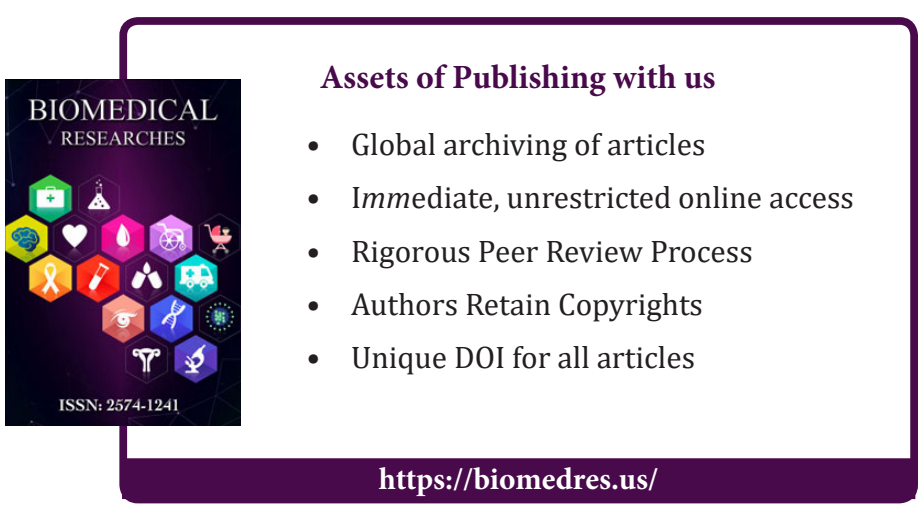

